\title{
Derivation of a Class of Frequency Distributions via Bayes's Theorem
}

\author{
By CARL-Erik Särndal
}

University of Michigan

[Received November 1964. Revised March 1965]

\begin{abstract}
SUMMARY
Being in some respects expository in character, this paper uses Bayes's theorem to derive a $k$-variate discrete probability distribution which is fundamental inasmuch as it proliferates, under various sets of asymptotic conditions, to yield as its limiting forms an array of other $k$-variate distributions including the multinomial, independent Poisson, independent negative binomial, independent gamma, Dirichlet and multivariate normal. A systematic arrangement of these distributions thereby suggests itself, and a number of properties pertaining to the distributions are discussed within the framework created by this arrangement.
\end{abstract}

\section{INTRODUCTION}

CONSIDER a model population the elements of which are known to classify into $k+1$ mutually exclusive classes, where $k \geqslant 1$ is a fixed integer. In this paper we find it convenient to think in terms of an urn containing balls of $k+1$ different colours, each colour representing a class. Denote the (unknown) number of balls of the $i$ th colour by $s_{i}(i=1, \ldots, k+1)$; the (known) total number of balls in the urn is $s=\sum_{i=1}^{k+1} s_{i}$. A first sample of balls (of fixed size $m$ ) has been drawn at random and without replacement, and the observed frequencies of the various colours are $m_{i}(i=1, \ldots, k+1)$, and $m=\Sigma_{1}^{k+1} m_{i}$. From the remainder population a second random sample of balls (of fixed size $n$ ) is drawn without replacement, and the observed frequencies are $n_{i}(i=1, \ldots, k+1)$, and $n=\Sigma_{1}^{k+1} n_{i}$. Denote the frequency vectors by $\mathbf{s}=\left(s_{1}, \ldots, s_{k}\right), \mathbf{m}=\left(m_{1}, \ldots, m_{k}\right), \mathbf{n}=\left(n_{1}, \ldots, n_{k}\right)$. Corresponding to these observed vectors there are vector random variables

$$
\mathbf{S}=\left(S_{1}, \ldots, S_{k}\right), \mathbf{M}=\left(M_{1}, \ldots, M_{k}\right), \mathbf{N}=\left(N_{1}, \ldots, N_{k}\right),
$$

respectively. (The sum of the $k+1$ frequencies being a fixed integer, it is sufficient to take only $k$ of them into account when defining the vector. Here we choose to consider the frequency of the $(k+1)$ th class as redundant.) For brevity we shall throughout the paper use a notation such that, for instance, $P(\mathbf{n} \mid \mathbf{m}, \mathbf{s})$ represents the probability that $\mathbf{N}=\mathbf{n}$ given $\mathbf{M}=\mathbf{m}$ and $\mathbf{S}=\mathbf{s}$. Also, throughout the paper, capital letters will denote random variables, and their small counterparts will denote observed values.

We shall investigate the distribution of the class frequency vector $\mathbf{N}$ of the second sample utilizing the information available to us by the fact that a first sample has previously been drawn, and that thereby we have observed the vector $\mathbf{M}=\mathbf{m}$. In such a situation the first sample represents, literally speaking, our prior knowledge of the population. The distribution of $\mathbf{N}$ will, however, also be influenced by the 
subjective choice of a prior distribution for $\mathbf{S}$. Two prior distributions which are later referred to as the Bose-Einstein prior and the Maxwell-Bolzmann prior are to be considered.

Attention will be focused on the case where the Bose-Einstein prior is used. This prior distribution is a uniform one, and it produces a distribution of $\mathbf{N}$ (given $\mathbf{M}=\mathbf{m}$ ) which has as its limiting forms, under various assumptions on the quantities $m, m_{i}$, $n$ and $n_{i}$, a spectrum of well-known distributions including the multinomial, Poisson, negative binomial, gamma, Dirichlet and multivariate normal distributions. The various assumptions made on $m, m_{i}, n$ and $n_{i}$, suggest a systematic arrangement of these distributions. Sections 3, 4 and 5 are devoted to developing this system and to discussing a number of properties relating to the distributions, and the way in which these properties fit into the system.

\section{The Joint Distribution of $\mathbf{S}, \mathbf{M}$ and $\mathbf{N}$}

Without specifying its form for the moment, let $P(\mathbf{s})$ denote the prior distribution of S. By elementary probability laws,

where

$$
P(\mathbf{m} \mid \mathbf{s})=\prod_{i=1}^{k+1}\left(\begin{array}{l}
s_{i} \\
m_{i}
\end{array}\right) /\left(\begin{array}{l}
s \\
m
\end{array}\right)
$$

$$
0 \leqslant m_{i} \leqslant m \quad(i=1, \ldots, k), \quad \sum_{i=1}^{k} m_{i} \leqslant m,
$$

and

where

$$
P(\mathbf{n} \mid \mathbf{m}, \mathbf{s})=\prod_{i=1}^{k+1}\left(\begin{array}{c}
s_{i}-m_{i} \\
n_{i}
\end{array}\right) /\left(\begin{array}{c}
s-m \\
n
\end{array}\right)
$$

$$
0 \leqslant n_{i} \leqslant n \quad(i=1, \ldots, k), \quad \sum_{i=1}^{k} n_{i} \leqslant n .
$$

(Note that some outcomes $\mathbf{m}$ for which (2.1) holds may actually be impossibilities, i.e. $P(\mathbf{m} \mid \mathbf{s})=0$. Likewise, for some outcomes $\mathbf{n}$ for which (2.2) holds we may have $P(\mathbf{n} \mid \mathbf{m}, \mathbf{s})=0$. In such cases the probability expressions would contain one or more binomial factors $\left(\begin{array}{l}a \\ b\end{array}\right)$ with $b>a$, which are to be interpreted as zero.)

We find

$$
\begin{aligned}
& P(\mathbf{s}, \mathbf{m}, \mathbf{n})=P(\mathbf{s}) P(\mathbf{m} \mid \mathbf{s}) P(\mathbf{n} \mid \mathbf{m}, \mathbf{s}) \\
& \quad=P(\mathbf{s}) \prod_{i=1}^{k+1}\left(\begin{array}{c}
s_{i} \\
m_{i}+n_{i}
\end{array}\right) /\left(\begin{array}{c}
s \\
m+n
\end{array}\right) \prod_{i=1}^{k+1}\left(\begin{array}{c}
m_{i}+n_{i} \\
n_{i}
\end{array}\right) /\left(\begin{array}{c}
m+n \\
n
\end{array}\right),
\end{aligned}
$$

where, in addition to (2.1) and (2.2),

$$
m_{i}+n_{i} \leqslant s_{i} \leqslant s \quad(i=1, \ldots, k), \quad \sum_{i=1}^{k} s_{i} \leqslant s .
$$

We recall that $s, m$ and $n$ are fixed integers, and they remain so throughout the paper.

At this point we shall make some assumption as to the particular form of the prior distribution, $P(\mathbf{s})$, and then proceed to find the conditional distribution of $\mathbf{N}$, given $\mathbf{M}=\mathbf{m}$. 


\section{Bose-Einstein Prior Distribution}

Consider $s$ indistinguishable balls, and assume that $k+1$ different colours are available. Let each of the $s$ balls be assigned one of the colours at random. Letting $s_{i}$ denote the frequency of balls of the $i$ th colour resulting from this procedure, the number of different frequency configurations $\mathbf{s}=\left(s_{1}, \ldots, s_{k}\right)$ that can arise is $\left(\begin{array}{c}s+k \\ k\end{array}\right)$. Under Bose-Einstein statistics (see, for example, Feller, 1957, p. 38) all the configurations are equally likely. Thus, the probability of any configuration is given by

for

$$
P(\mathbf{s})=1 /\left(\begin{array}{c}
s+k \\
k
\end{array}\right)
$$

$$
0 \leqslant s_{i} \leqslant s \quad(i=1, \ldots, k), \quad \sum_{i=1}^{k} s_{i} \leqslant s .
$$

The distribution (3.1) will be referred to as a Bose-Einstein prior for $\mathbf{S}$.

Inserting (3.1) into (2.3) we obtain

$$
P(\mathbf{s}, \mathbf{m}, \mathbf{n})=\prod_{i=1}^{k+1}\left(\begin{array}{c}
s_{i} \\
m_{i}+n_{i}
\end{array}\right) /\left(\begin{array}{c}
s+k \\
m+n+k
\end{array}\right) \prod_{i=1}^{k+1}\left(\begin{array}{c}
m_{i}+n_{i} \\
n_{i}
\end{array}\right) / \frac{(m+n+k) !}{m ! n ! k !} .
$$

We eliminate $s$ by summing over (2.4), whereupon the first product expression becomes unity, yielding

The marginal distributions are

$$
P(\mathbf{m}, \mathbf{n})=\prod_{i=1}^{k+1}\left(\begin{array}{c}
m_{i}+n_{i} \\
n_{i}
\end{array}\right) / \frac{(m+k+n) !}{m ! k ! n !} .
$$

$$
P(\mathbf{m})=1 /\left(\begin{array}{c}
m+k \\
k
\end{array}\right), \quad P(\mathbf{n})=1 /\left(\begin{array}{c}
n+k \\
k
\end{array}\right),
$$

defined over (2.1) and (2.2), respectively. Thus, a Bose-Einstein prior for $\mathbf{S}$ produces marginal distributions of the Bose-Einstein type for $\mathbf{M}$ and $\mathbf{N}$. However, $\mathbf{M}$ and $\mathbf{N}$ are not independent, and the conditional distribution of $\mathbf{N}$ is found to be

$$
P(\mathbf{n} \mid \mathbf{m})=\prod_{i=1}^{k+1}\left(\begin{array}{c}
m_{i}+n_{i} \\
n_{i}
\end{array}\right) /\left(\begin{array}{c}
m+k+n \\
n
\end{array}\right),
$$

defined over (2.2). This distribution was also discussed in Särndal (1964), although in a somewhat different context. A univariate version of (3.3) is given, with different notation in Fisher (1956, p. 111).

The distribution (3.3) has a number of limiting distributions which are arrived at under varying assumptions on $m, m_{i}, n$ and $n_{i}(i=1, \ldots, k+1)$. We introduce the following notation for the distribution (3.3) and its limiting forms. Let

$$
A=\prod_{i=1}^{k+1}\left(\begin{array}{c}
m_{i}+n_{i} \\
n_{i}
\end{array}\right) /\left(\begin{array}{c}
m+k+n \\
n
\end{array}\right)
$$

be the $k$-variate distribution (3.3). As a matter of convenience we shall refer to the integers $m_{i}+1(i=1, \ldots, k+1)$, rather than to the $m_{i}$ themselves, as the parameters of this distribution. We may also consider

$$
m+k+1=\sum_{i=1}^{k+1}\left(m_{i}+1\right)
$$


as a parameter. The random variables $N_{i}$ take on values $n_{i}$ which are restricted by (2.2). The first two moments are

$$
\begin{aligned}
E\left(N_{i} \mid \mathbf{m}\right) & =n \pi_{i}, \\
\operatorname{var}\left(N_{i} \mid \mathbf{m}\right) & =n^{2}\left(1+\frac{m+k+1}{n}\right) \frac{\pi_{i}\left(1-\pi_{i}\right)}{m+k+2}, \\
\operatorname{cov}\left(N_{i}, N_{j} \mid \mathbf{m}\right) & =-n^{2}\left(1+\frac{m+k+1}{n}\right) \frac{\pi_{i} \pi_{j}}{m+k+2},
\end{aligned}
$$

where

$$
\pi_{i}=\left(m_{i}+1\right) /(m+k+1) .
$$

Let

$$
B^{\prime}=\left(n ! / \prod_{i=1}^{k+1} n_{i} !\right) \prod_{i=1}^{k+1} p_{i}^{n_{i}}
$$

be the $k$-variate multinomial distribution where $n$ and $p_{i}(i=1, \ldots, k+1)$ are parameters such that

$$
0<p_{i}<1 ; \quad \sum_{i=1}^{k+1} p_{i}=1 .
$$

The distribution is defined for non-negative integers $n_{i}$ fulfilling (2.2).

Let

$$
B_{1}=\left\{(m+k) ! / \prod_{i=1}^{k+1} m_{i} !\right\} \prod_{i=1}^{k+1} y_{i}^{m_{i}}
$$

denote the $k$-variate Dirichlet distribution. The integers $m_{i}+1(i=1, \ldots, k+1)$ are called the parameters of the distribution which is defined over the $k$-dimensional simplex

$$
0 \leqslant y_{i} \leqslant 1 \quad(i=1, \ldots, k), \quad \sum_{i=1}^{k} y_{i} \leqslant 1 .
$$

Let

$$
A_{0}=\prod_{i=1}^{k}\left(\begin{array}{c}
m_{i}+n_{i} \\
n_{i}
\end{array}\right) \lambda^{m_{i}+1}(1-\lambda)^{n_{i}}
$$

be the joint probability of $k$ independent discrete random variables $N_{i}(i=1, \ldots, k)$, each having a negative binomial distribution with the same parameter $\lambda$, where $0<\lambda<1$, but possibly differing $m_{i}$. Again we shall refer to $m_{i}+1$ (rather than to $m_{i}$ ) as a parameter. The variables $N_{i}$ take on values $n_{i}$ restricted by

$$
0 \leqslant n_{i}<\infty \quad(i=1, \ldots, k) .
$$

Let

$$
C^{\prime}=\prod_{i=1}^{k} \frac{\theta_{i}^{n_{i}}}{n_{i} !} e^{-\theta_{i}}
$$

denote the joint probability of $k$ independent discrete random variables

$$
N_{i} \quad(i=1, \ldots, k),
$$


each having a Poisson distribution with parameter $\theta_{i}>0(i=1, \ldots, k)$. The $n_{i}$ are subject to (3.5).

Let

$$
C_{1}=\prod_{i=1}^{k} \frac{x_{i}^{m_{i}}}{m_{i} !} e^{-x_{i}}
$$

be the joint density of $k$ independent continuous random variables $X_{i}(i=1, \ldots, k)$, each having a gamma distribution with parameter $m_{i}+1$. The range of $X_{i}$ is $0<x_{i}<\infty(i=1, \ldots, k)$.

Let

$$
D=(2 \pi)^{-\frac{1}{2} k}|\mathbf{R}|^{\frac{1}{2}} \exp \left\{-\frac{1}{2}(\mathbf{z}-\boldsymbol{\mu}) \mathbf{R}(\mathbf{z}-\boldsymbol{\mu})^{\prime}\right\}
$$

be the $k$-variate normal density for the random vector $Z=\left(Z_{1}, \ldots, Z_{k}\right)$, where $\boldsymbol{\mu}=E(\mathbf{Z})=\left(\mu_{1}, \ldots, \mu_{k}\right)$ is the mean vector and $\mathbf{R}^{-1}=\mathbf{V}$ is the $k \times k$ variancecovariance matrix of $Z_{1}, \ldots, Z_{k}$ with elements $v_{i i}=\operatorname{var}\left(Z_{i}\right), v_{i j}=\operatorname{cov}\left(Z_{i}, Z_{j}\right)(i \neq j)$. In particular, if $v_{i j}=0(i \neq j)$, let

$$
D_{0}=\prod_{i=1}^{k}\left(2 \pi v_{i i}\right)^{-1} \exp \left\{-\frac{1}{2}\left(z_{i}-\mu_{i}\right)^{2} / v_{i i}\right\}
$$

be the joint density of $k$ independently distributed continuous random variables $Z_{i}$, each being normal $\left(\mu_{i}, v_{i i}\right)$.

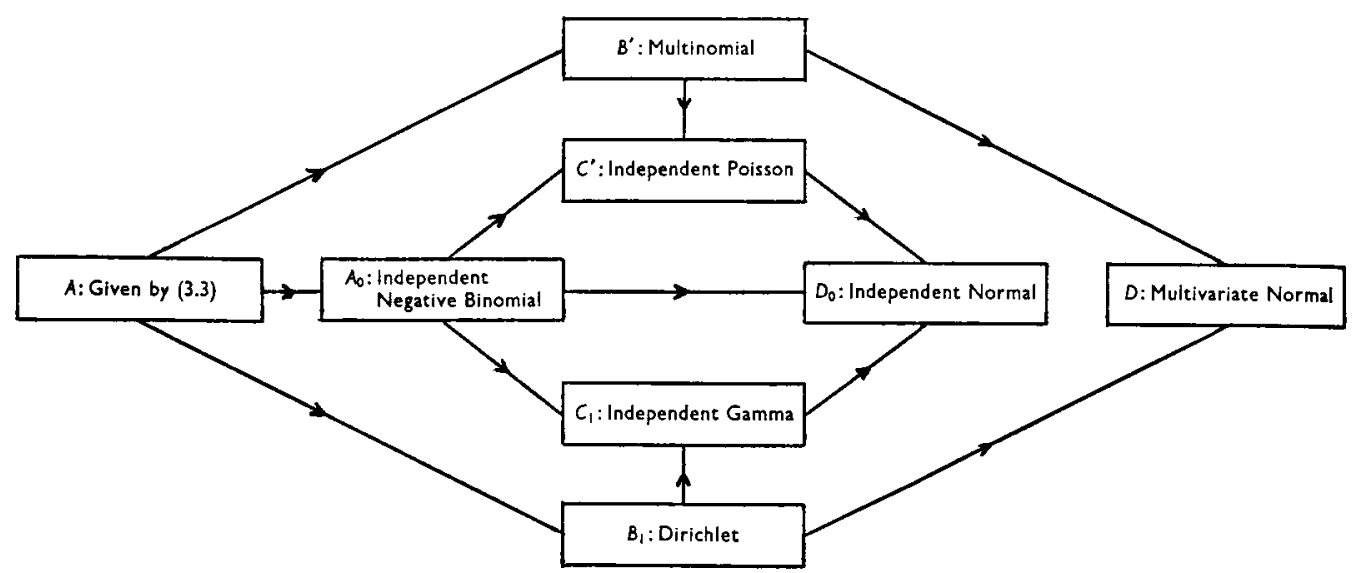

FIG. 1. Arrow scheme showing limiting relations between various $k$-variate distributions.

The first two moments of $B^{\prime}, B_{1}, A_{0}, C^{\prime}$ and $C_{1}$ are given in Table 2 . The distributions $A, B^{\prime}, B_{1}, A_{0}, C^{\prime}, C_{1}, D_{0}$ and $D$ are interconnected (in a certain limiting sense) in a way which is described by the arrow scheme shown in Fig. 1.

Under certain limiting conditions, the distribution of one box approaches (after possible multiplication by a constant) that of another box connected with the first one by a line and an arrow pointing toward the limit distribution. The proofs of all these relations are elementary, and need not be further discussed here, and, indeed, some of them provide standard material for many textbooks in elementary theoretical statistics. However, for completeness the exact nature of the limiting conditions represented by each arrow in Fig. 1 are listed in Tables 1 and 2. Table 1 also clarifies how the newly introduced parameters and random elements (i.e. $p_{i}, \lambda, y_{i}, \theta_{i}$ and $x_{i}$ ) depend in the limit upon the quantities $m, m_{i}, n$ and $n_{i}$. 


\section{TABLE 1}

Conditions for some limiting relations displayed in Fig. 1

\begin{tabular}{clll}
\hline No. & $\begin{array}{c}\text { Limiting } \\
\text { relation }\end{array}$ & \multicolumn{1}{c}{ Conditions } & \multicolumn{1}{c}{$\begin{array}{c}\text { Quantities remaining } \\
\text { finite }\end{array}$} \\
\hline 1 & $A \rightarrow B^{\prime}$ & $m \rightarrow \infty ; m_{i} / m \rightarrow p_{i}>0(i=1, \ldots, k+1)$ & $n, n_{i}(i=1, \ldots, k+1)$ \\
2 & $A \rightarrow A_{0}$ & $m, m_{k+1}, n, n_{k+1} \rightarrow \infty ; m /(m+n) \rightarrow \lambda>0$ & $m_{i}, n_{i}(i=1, \ldots, k)$ \\
3 & $n^{k} A \rightarrow B_{1}$ & $n \rightarrow \infty ; n_{i} / n \rightarrow y_{i}>0(i=1, \ldots, k+1)$ & $m, m_{i}(i=1, \ldots, k+1)$ \\
4 & $B^{\prime} \rightarrow C^{\prime}$ & $n, n_{k+1} \rightarrow \infty ; p_{i} \rightarrow 0 ; n p_{i} \rightarrow \theta_{i}>0(i=1, \ldots, k)$ & $n_{i}(i=1, \ldots, k)$ \\
5 & $A_{0} \rightarrow C^{\prime}$ & $m_{i} \rightarrow \infty(i=1, \ldots, k) ; \lambda \rightarrow 1 ; m_{i}(1-\lambda) / \lambda \rightarrow \theta_{i}>0(i=1, \ldots, k)$ & $n_{i}(i=1, \ldots, k)$ \\
6 & $\lambda^{-k} A_{0} \rightarrow C_{1}$ & $n_{i} \rightarrow \infty(i=1, \ldots, k) ; \lambda \rightarrow 0 ; n_{i} \lambda /(1-\lambda) \rightarrow x_{i}>0(i=1, \ldots, k)$ & $m_{i}(i=1, \ldots, k)$ \\
7 & $m^{-k} B_{1} \rightarrow C_{1}$ & $m, m_{k+1} \rightarrow \infty ; y_{i} \rightarrow 0 ; m y_{i} \rightarrow x_{i}>0(i=1, \ldots, k)$ & $m_{i}(i=1, \ldots, k)$ \\
\hline
\end{tabular}

\section{TABLE 2}

Conditions under which distributions $B^{\prime}, C^{\prime}, A_{0}, C_{1}$ and $B_{1}$ approach a multivariate normal distribution

\begin{tabular}{|c|c|c|c|c|c|}
\hline \multirow{2}{*}{$\begin{array}{l}\text { Limiting } \\
\text { relation }\end{array}$} & \multirow{2}{*}{ Conditions } & \multicolumn{4}{|c|}{ Elements of $\mathbf{z}, \mu$ and $\mathrm{V}(i, j=1, \ldots, k)$} \\
\hline & & $z_{i}$ & $\mu_{i}$ & $v_{i i}$ & $v_{i j}(i \neq j)$ \\
\hline$B^{\prime} \rightarrow D$ & $\begin{array}{l}n, n_{i} \rightarrow \infty(i=1, \ldots, k+1) ; \\
n \sum_{i=1}^{k+1} \frac{\left(n_{i} / n-p_{i}\right)^{3}}{p_{i}^{2}} \rightarrow 0\end{array}$ & $n_{i}$ & $n p_{i}$ & $n p_{i}\left(1-p_{i}\right)$ & $-n p_{i} p_{j}$ \\
\hline$C^{\prime} \rightarrow D_{0}$ & $n_{i} \rightarrow \infty, \frac{\left(n_{i}-\theta_{i}\right)^{3}}{\theta_{i}^{2}} \rightarrow 0(i=1, \ldots, k)$ & $n_{i}$ & $\theta_{i}$ & $\theta_{i}$ & $\mathbf{0}$ \\
\hline$A_{0} \rightarrow D_{0}$ & $\begin{aligned} & m_{i}, n_{i} \rightarrow \infty, \frac{\left\{n_{i}-\left(m_{i}+1\right)(1-\lambda) / \lambda\right\}^{3}}{\left(m_{i}+1\right)^{2}} \rightarrow 0 \\
&(i=1, \ldots, k)\end{aligned}$ & $n_{i}$ & $\frac{\left(m_{i}+1\right)(1-\lambda)}{\lambda}$ & $\frac{\left(m_{i}+1\right)(1-\lambda)}{\lambda^{2}}$ & 0 \\
\hline$C_{1} \rightarrow D_{0}$ & $m_{i} \rightarrow \infty, \frac{\left\{x_{i}-\left(m_{i}+1\right)\right\}^{3}}{\left(m_{i}+1\right)^{2}} \rightarrow 0(i=1, \ldots, k)$ & $x_{i}$ & $m_{i}+1$ & $m_{i}+1$ & 0 \\
\hline$B_{1} \rightarrow D$ & $\begin{array}{l}m, m_{i} \rightarrow \infty(i=1, \ldots, k+1) \\
\quad(m+k+2) \sum_{i=1}^{k+1} \frac{\left(y_{i}-\pi_{i}\right)^{3}}{\pi_{i}^{2}} \rightarrow 0\end{array}$ & $y_{i}$ & $\pi_{i}$ & $\frac{\pi_{i}\left(1-\pi_{i}\right)}{m+k+2}$ & $-\frac{\pi_{i} \pi_{j}}{m+k+2}$ \\
\hline
\end{tabular}

In this table, $\pi_{i}$ is given by (3.4). 


\section{A Discussion of the Distributions in Fig. 1}

A number of observations are readily made by studying the arrow scheme of Fig. 1 and the distributions associated with it.

(1) The set of distributions can be divided into four subsets each containing two distributions, the upper set $\left(B^{\prime}, C^{\prime}\right)$, the lower set $\left(C_{1}, B_{1}\right)$, the left-hand set $\left(A, A_{0}\right)$, and the right-hand set $\left(D_{0}, D\right)$, such that the distributions of each subset are similar with respect to the behaviour of the quantities $m_{i}$ and $n_{i}(i=1, \ldots, k)$. For the lefthand set, the $m_{i}$ and the $n_{i}$ are finite, while for the right-hand set the $m_{i}$ as well as the $n_{i}$ are infinite. The upper set refers to the situation where the $m_{i}$ are infinite but the $n_{i}$ are finite, whereas for the lower set the $n_{i}$ are infinite and the $m_{i}$ finite.

(2) The distributions can be divided into an "outer set" consisting of $A, B^{\prime}, B_{1}$ and $D$ and an "inner set" consisting of $A_{0}, C^{\prime}, C_{1}$ and $D_{0}$. The common characteristic of the outer set is the fact that its members are joint distributions of dependent random variables, whereas the inner set is made up of joint distributions of independent random variables.

Under (3) to (7) below, as well as in Section 5, a number of properties relating to the distributions of Fig. 1 are discussed. The majority of these properties are well known and often cited, and our main concern is to observe how they fit into the pattern set by Fig. 1.

Property (3) deals with the pair $B^{\prime}, B_{1}$, of the outer set, while (4) brings forth a corresponding property pertaining to the pair $C^{\prime}, C_{1}$ of the inner set.

(3) $B^{\prime}$ and $B_{1}$ are "mirror images" of each other in the following sense. If the $N_{i}$ (given $P_{i}=p_{i}$ ) have the multinomial distribution $B^{\prime}$, and the parameters $p_{i}$ are considered random variables $P_{i}$ possessing a uniform prior distribution, then the posterior distribution of the $P_{i}$ is a $k$-variate Dirichlet distribution of the type $B_{1}$. Conversely, if the variables $Y_{i}$ (given $M_{i}=m_{i}$ ) have the distribution $B_{1}$ and the $M_{i}$ are assumed to have a uniform prior, then their posterior distribution is of the type $B^{\prime}$.

(4) Similarly, $C^{\prime}$ and $C_{1}$ are mirror images of each other. If $N_{i}$ has a Poisson distribution with parameter $\theta_{i}$, which is considered to be random having a uniform prior distribution, then the posterior distribution of this parameter is a gamma density. Or, conversely, if $X_{i}$ (given the parameter $M_{i}+1=m_{i}+1$ ) has a gamma distribution, and $M_{i}$ is assumed to be random with a uniform prior, then the posterior distribution of $M_{i}$ is Poisson.

Property (5) relates to the distributions of the outer set, while its counterpart for the inner set is stated as property (6).

(5) Combining $B^{\prime}$ and $B_{1}$ in a certain way leads to $A$. Let the distribution of the $N_{i}$ (given $P_{i}=p_{i}$ ) be given by $B^{\prime}$. Assume that the parameters $P_{i}$ are of random nature having (prior) density $B_{1}$ with parameters $m_{i}+1(i=1, \ldots, k+1)$. Then the (marginal) joint density of the $N_{i}$ is given by $A$. Furthermore, the posterior density of the $P_{i}$ (after having observed the $n_{i}$ ) is a density $B_{1}$ with parameters

$$
m_{i}+n_{i}+1(i=1, \ldots, k+1) \text {. }
$$

(In a Bayesian's language, the kernel of the prior density combines with the sample kernel in the same way that two sample kernels would combine; see Raiffa and Schlaifer (1961).)

(6) Combining $C^{\prime}$ and $C_{1}$ in a similar way leads to $A_{0}$. If $N_{i}$ has a Poisson distribution with parameter $T_{i}=\theta_{i}$ and this parameter is considered to be random such that $T_{i} / \gamma$ (where $\gamma>0$ is an arbitrary scale factor) has a (prior) gamma density with parameter $m_{i}+1$, then the (marginal) distribution of $N_{i}$ is negative binomial with 
parameters $m_{i}+1$ and $\lambda=1 /(1+\gamma)$. Furthermore, the posterior density of $T_{i} / \gamma$ is a gamma density with parameter $m_{i}+1+n_{i}$, a convolution corresponding to the one mentioned in (5).

Property (7) illustrates a relationship by which members of the inner set of $k$-variate distributions are turned into $(k-1)$-variate distributions belonging to the outer set.

(7) Given that the sum of the $k$ variables in $A_{0}, C^{\prime}$ and $C_{1}$ is fixed, the joint density of the resulting $k-1$ non-redundant variables is given by $(k-1)$-variate correspondences of $A, B^{\prime}$ and $B_{1}$, respectively. We can express this in more specific terms as $(7 \mathrm{a})-(7 \mathrm{c})$, where

$$
m^{\prime}=\sum_{i=1}^{k} m_{i}, \quad \theta^{\prime}=\sum_{i=1}^{k} \theta_{i}
$$

(7a) If the $N_{i}(i=1, \ldots, k)$ have the distribution $A_{0}$, and given that $\Sigma_{1}^{k} N_{i}=N^{\prime}$ is fixed at, say, $N^{\prime}=n^{\prime}$, then $N_{1}, \ldots, N_{k-1}$ are distributed according to a $(k-1)$-variate version of $\boldsymbol{A}$ with parameters $m_{i}+1(i=1, \ldots, k)$, i.e.

$$
\prod_{i=1}^{k}\left(\begin{array}{c}
m_{i}+n_{i} \\
n_{i}
\end{array}\right) /\left(\begin{array}{c}
m^{\prime}+k-1+n^{\prime} \\
n^{\prime}
\end{array}\right) \text {. }
$$

(7b) If the variables $N_{i}(i=1, \ldots, k)$ are jointly distributed according to $C^{\prime}$, and given that $\Sigma_{1}^{k} N_{i}=N^{\prime}$ equals $n^{\prime}$, then the resulting joint distribution of $N_{1}, \ldots, N_{k-1}$ is a $(k-1)$-variate version of $\boldsymbol{B}^{\prime}$, namely the $(k-1)$-variate multinomial distribution with parameters $n^{\prime}, \theta_{i} / \theta^{\prime}(i=1, \ldots, k)$.

(7c) If the variables $X_{i}(i=1, \ldots, k)$ are jointly distributed according to $C_{1}$, and $\Sigma_{1}^{k} X_{i}=X^{\prime}$ is held fixed, then the joint density of the variables $X_{i} / X^{\prime}(i=1, \ldots, k-1)$ is a $(k-1)$-variate distribution of the type $B_{1}$, namely, a $(k-1)$-variate Dirichlet distribution with parameters $m_{i}+1(i=1, \ldots, k) ; m^{\prime}+k=\Sigma_{1}^{k}\left(m_{i}+1\right)$.

\section{AdDitive Properties}

A number of well-known additive (or convolution) properties relating to the multinomial, Poisson, negative binomial, gamma and Dirichlet distributions are considered in this Section. They can all be viewed in the light of an additivity property that pertains to the distribution (3.3).

Consider the set of integers $U=\{1,2, \ldots, k+1\}$, and let $q$ be an integer such that $1 \leqslant q \leqslant k$. Furthermore, consider an arbitrary division of $U$ into $q+1$ mutually exclusive non-empty subsets $T_{1}, \ldots, T_{q+1}$. Let

$$
\sum_{i \in T_{j}} m_{i}=m_{T_{j}}, \quad \sum_{i \in T_{j}} n_{i}=n_{T}
$$

be the class frequencies for a new system of $q+1$ classes which is obtained by pooling those classes $i$ for which $i \in T_{j}(j=1, \ldots, q+1)$. Each of the new classes is then comprehending one or more of the old ones. Denote the number of elements in $T_{j}$ by $t_{j}$. We have

$$
t_{j} \geqslant 1 \quad(j=1, \ldots, q+1) ; \quad \sum_{j=1}^{q+1} t_{j}=k+1
$$


Let

$$
\mathbf{m}_{T}=\left(m_{T_{1}}, \ldots, m_{T_{q+1}}\right), \quad \mathbf{n}_{T}=\left(n_{T_{1}}, \ldots, n_{T_{\mathbf{q}+1}}\right),
$$

be the observed vectors of class frequencies for the new system of classes, and let $\mathbf{M}_{T}$ and $\mathbf{N}_{T}$ be the corresponding vector random variables. The distribution of $\mathbf{N}_{T}$ given $\mathbf{M}_{T}=\mathbf{m}_{T}$ is (under the assumption of a Bose-Einstein prior) obtained from (3.3) as a $q$-variate version of $A$, namely

$$
P\left(\mathbf{n}_{T} \mid \mathbf{m}_{T}\right)=\prod_{j=1}^{q+1}\left(\begin{array}{c}
m_{T_{j}}+t_{j}-1+n_{T_{j}} \\
n_{T_{j}}
\end{array}\right) /\left(\begin{array}{c}
m+k+n \\
n
\end{array}\right),
$$

the parameters being $m_{T}+t_{j}(j=1, \ldots, q+1)$.

We shall consider this additive property in more detail for the case $q=1$, and in this connection we introduce some notation that will be used in the remainder of this Section. Let $T$ be an arbitrary subset of $U$ containing $t$ elements. Let $i_{0}$ be an arbitrary element in $T$, and let $i_{1}$ be an arbitrary element in the complement set $\bar{T}=U-T$. Also, let

$$
\begin{aligned}
T^{\prime} & =T-i_{0}, \quad \bar{T}^{\prime}=\bar{T}-i_{1}, \\
n_{T} & =\sum_{i \in T} n_{i}, \quad m_{T}=\sum_{i \in T} m_{i} .
\end{aligned}
$$

The distribution of

$$
N_{T}=\sum_{i \in T} N_{i}
$$

(given $M_{T}=m_{T}$ ) is then obtained as

$$
\Sigma P(\mathbf{n} \mid \mathbf{m})=P\left(n_{T} \mid m_{T}\right),
$$

where the summation is a multiple one extending over all non-negative $n_{i}\left(i \in T^{\prime}+\bar{T}^{\prime}\right)$ such that

$$
\sum_{i \in T^{\prime}} n_{i} \leqslant n_{T}, \quad \sum_{i \in \bar{T}^{\prime}} n_{i} \leqslant n-n_{T}
$$

The distribution of $N_{T}$ (given $M_{T}=m_{T}$ ) is denoted by $E$, say, and is given by

$$
E=P\left(n_{T} \mid m_{T}\right)=\left(\begin{array}{c}
m_{T}+t-1+n_{T} \\
n_{T}
\end{array}\right)\left(\begin{array}{c}
m-m_{T}+k-t+n-n_{T} \\
n-n_{T}
\end{array}\right) /\left(\begin{array}{c}
m+k+n \\
n
\end{array}\right)
$$

for $0 \leqslant n_{T} \leqslant n$. It is, of course, a special case of (5.1).

Consider again equation (5.2) and let us apply to both members those limiting conditions (as stated in Table 1) that turned the distribution $P(\mathbf{n} \mid \mathbf{m})=A$ appearing in the left member into a $k$-variate multinomial, independent Poisson, independent negative binomial, independent gamma or Dirichlet distribution. (In Table 1 these conditions are found under (1), (4), (2), (7) and (3), respectively.) Letting these limiting conditions operate one by one on the right member of (5.2), i.e. on the distribution of $N_{T}$, we obtain in the limit a binomial, Poisson, negative binomial, gamma or beta distribution, respectively. Using the definitions of $p_{i}, \lambda, y_{i}, \theta_{i}$ and $x_{i}$ introduced in Table 1, the limiting forms of $E$ given by (5.3) turn out to be as follows (the figure in 
parentheses refers to the relation number in Table 1 under which the limiting conditions are stated).

(3) $\frac{(m+k) !}{\left(m_{T}+t-1\right) !\left(m-m_{T}+k-t\right) !} y_{T}^{m_{T}+l-1}\left(1-y_{T}\right)^{m-m_{T}+k-\imath} ; \quad y_{T}=\lim \frac{n_{T}}{n}=\sum_{i \in T} y_{i}$.

(Note that $n E$, rather than $E$ itself, approaches the beta density of the last line. If this beta density is denoted by $E_{1}$, then $m^{-1} E_{1}$ approaches the gamma density associated with condition (7). In these two cases of continuous random variables, the summation in (5.2) becomes an integration over two simplexes of dimensions $t-1$ and $k-t$, respectively, after the limiting processes have been performed.)

A number of additive properties are thereby illustrated. For a sum of independent random variables $N_{i}$ or $X_{i}(i \in T)$ these are as follows.

(A1) If each $N_{i}$ is Poisson distributed with parameter $\theta_{i}$, then $\Sigma N_{i}$ is Poisson distributed with parameter $\Sigma \theta_{i}$.

(A2) If each $N_{i}$ is distributed according to a negative binomial distribution with parameters $\lambda$ and $m_{i}+1$, then $\Sigma N_{i}$ has a negative binomial distribution with parameters $\lambda$ and $\Sigma\left(m_{i}+1\right)$.

(A3) If each $X_{i}$ is gamma distributed with parameter $m_{i}+1$, then $\Sigma X_{i}$ is gamma distributed with parameter $\Sigma\left(m_{i}+1\right)$.

For a sum of dependent random variables $N_{i}$ or $Y_{i}(i \in T)$, we have the following additive properties:

(B1) If the joint distribution of the $N_{i}$ is multinomial such that the marginal distribution of each $N_{i}$ is binomial with parameters $n, p_{i}$, then $\Sigma N_{i}$ is binomial with parameters $n, \Sigma p_{i}$.

(B2) If the joint distribution of the $Y_{i}$ is a Dirichlet distribution such that the marginal distribution of each $Y_{i}$ is a beta density with parameters $m_{i}+1$, $m+k+1-\left(m_{i}+1\right)$, i.e. the density is

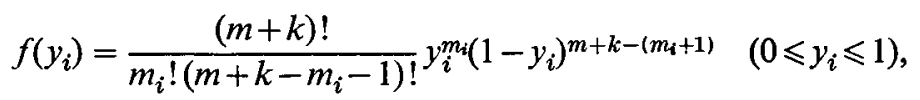

then $\Sigma Y_{i}$ has a beta distribution with parameters $\Sigma\left(m_{i}+1\right), m+k+1-\Sigma\left(m_{i}+1\right)$.

Finally, by the connections with the normal distributions brought forth by Fig. 1, it is clear that similar additive properties can be formulated for a sum of normally distributed random variables.

\section{Maxwell-Bolzmann Prior Distribution}

This final Section gives one brief illustration of a situation in which a non-uniform prior is chosen for $\mathbf{S}$. 
Consider $s$ balls that are distinguishable so that, for instance, each ball is labelled with an integer from 1 to $s$. Each of the balls is assigned at random one out of $k+1$ different colours. The number of different assignments is $(k+1)^{s}$, and, under Maxwell-Bolzmann statistics (see Feller, 1957, p. 38) all of them are equally likely. If $s_{i}$ is the number of balls having the $i$ th colour, the probability of the frequency configuration $\mathbf{s}=\left(s_{1}, \ldots, s_{k}\right)$ then becomes

$$
P(\mathbf{s})=\left(s ! / \prod_{i=1}^{k+1} s_{i} !\right)(k+1)^{-s}
$$

defined over (3.2). This we shall call a Maxwell-Bolzmann prior; clearly it is a multinomial with parameters $s$ and equal $p_{i}=1 /(k+1)(i=1, \ldots, k+1)$. Using it as a prior distribution for $\mathbf{S}$ in (2.3), we find

$$
P(\mathbf{s}, \mathbf{m}, \mathbf{n})=\frac{(s-m-n) !}{\prod_{i=1}^{k+1}\left(s_{i}-m_{i}-n_{i}\right) ! \prod_{i=1}^{k+1} m_{i} !} \frac{m !}{\prod_{i=1}^{k+1} n_{i} !}(k+1)^{-s}
$$

Eliminating $s$ by summing over (2.4), we obtain

$$
P(\mathbf{m}, \mathbf{n})=\frac{m !}{\prod_{i=1}^{k+1} m_{i} !} \frac{n !}{\prod_{i=1}^{k+1} n_{i} !}(k+1)^{-(m+n)} .
$$

Consequently $\mathbf{M}$ and $\mathbf{N}$ are independently distributed random vectors, each having a Maxwell-Bolzmann type marginal distribution. In particular,

$$
P(\mathbf{n})=P(\mathbf{n} \mid \mathbf{m})=\left(n ! / \prod_{i=1}^{k+1} n_{i}\right)(k+1)^{-n},
$$

i.e. a multinomial distribution with parameters $n$ and $p_{i}=1 /(k+1)(i=1, \ldots, k+1)$.

Choosing a Maxwell-Bolzmann prior distribution for $\mathbf{S}$ thus results in a situation in which the first sample does not provide any information towards the outcome of the second sample.

\section{ACKNOWLEDGEMENT}

This research was supported by the United States Army Research Office, Durham, North Carolina.

\section{REFERENCES}

Feller, W. (1957), An Introduction to Probability Theory and Its Applications, Vol. I, 2nd ed. New York: Wiley.

Fisher, R. A. (1956), Statistical Methods and Scientific Inference. Edinburgh: Oliver and Boyd.

Raiffa, H. and Schlaifer, R. (1961), Applied Statistical Decision Theory. Boston: Harvard University Graduate School of Business Administration.

SärNDAL, C-E. (1964). "A unified derivation of some nonparametric distributions", J. Amer. statist. Ass., 59, 1042-53. 